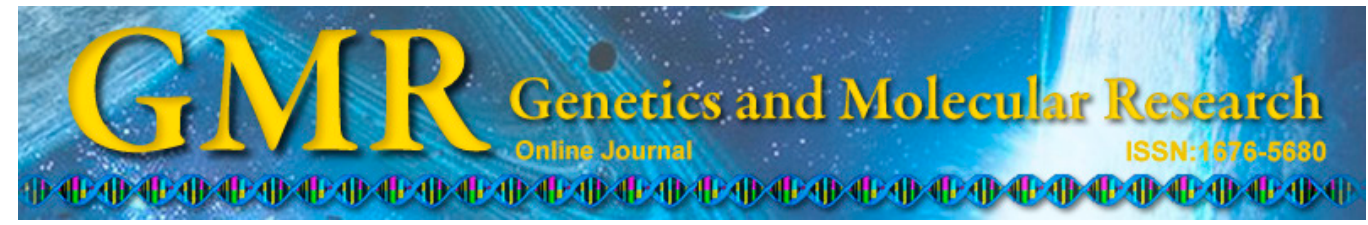

\title{
Single nucleotide polymorphism in the RECQL5 gene increased osteosarcoma susceptibility in a Chinese Han population
}

\author{
Y.Z. Dong, Y.X. Huang and T. Lu \\ Department of Orthopedics, \\ The First Affiliated Hospital of Xinxiang Medical College, Weihui, China
}

Corresponding author: Y.Z. Dong

E-mail: dongy_zhen@yeah.net

Genet. Mol. Res. 14 (1): 1899-1902 (2015)

Received February 25, 2014

Accepted September 7, 2014

Published March 13, 2015

DOI http://dx.doi.org/10.4238/2015.March.13.18

\begin{abstract}
In this study, we investigated the association between a RECQL genetic polymorphism and osteosarcoma in a Chinese population. We selected rs 820196 in the RECQL5 gene and genotyped 185 patients with osteosarcoma and 201 age- and gender-matched noncancer controls. We found that the $\mathrm{CC}$ genotype was more frequent in the osteosarcoma group compared to the control group $(\mathrm{P}=0.011)$. We also found that the $\mathrm{C}$ allele was more common in osteosarcoma patients than that in control subjects $(\mathrm{P}=0.004)$. Our results suggested that the RECQL5 genetic polymorphism was associated with osteosarcoma in a Chinese population.
\end{abstract}

Key words: Osteosarcoma; Polymorphism; RECQL5 


\section{INTRODUCTION}

Osteosarcoma (OS) is the most common malignancy in bone and is most frequently observed in children and young adults (Dorfman and Czerniak, 1995). The exact mechanisms of osteosarcoma are largely unknown, but many studies have suggested that multiple genetic and environmental factors play important roles in OS carcinogenesis. Patients with localized OS at presentation have a $60-80 \%$ rate of long-term survival, while metastatic disease carries a poorer prognosis (Kager et al., 2003; Mirabello et al., 2009). Previous studies suggested that OS is a complex disease resulting from the interaction between environmental factors and genetics (Zhou et al., 2014; Wang et al., 2014).

Recently, studies have indicated that DNA damage leads to genomic instability, cancer transformation, or cell death (Bohr, 2008). The $R E C Q$ family is a highly conserved group of DNA helicases required to maintain genome stability and integrity, which plays important roles in DNA replication and repair pathways including mismatch repair, nucleotide excision repair, and direct repair (Sharma et al., 2006; Hu et al., 2007). Therefore, RECQL5 has been reported to be associated with several cancers, and genetic polymorphisms may increase the risk of human cancers (Hu et al., 2007; Pellatt et al., 2012). RECQL5 is one member of the RECQL family. The relationship between the polymorphism and OS is not understood. In the present study, we investigated the association between RECQL5 gene polymorphisms and OS in a Chinese population.

\section{MATERIAL AND METHODS}

\section{Study population}

Written informed consent was obtained from each participant. The study protocol was approved by the Ethics Committee of the First Affiliated Hospital of Xinxiang Medical College. A total of 185 patients diagnosed with OS (7-44 years of age) and 201 healthy subjects (10-45 years of age) were included in the present study. All subjects were recruited from the First Affiliated Hospital of Xinxiang Medical College between March 2001 and March 2012. The cancer-free controls were matched with OS patients with regards to gender, age, and residence area.

\section{Genotyping}

Genomic DNA was extracted from peripheral blood leukocytes using a DNA extraction Kit (Beijing Bioteke Co. Ltd., Beijing, China). Genotyping was confirmed by TaqMan method as described previously (Li et al., 2009).

\section{Statistical analyses}

All statistical analyses were conducted using the Statistical Package for Social Sciences software (SPSS, Windows version release 15.0; SPSS Inc., Chicago, IL, USA). Hardy-Weinberg equilibrium in allele and genotype distribution was assessed by using the chisquared $\left(\chi^{2}\right)$ test. The odds ratios $(\mathrm{ORs})$ and $95 \%$ confidence intervals $(95 \% \mathrm{CIs})$ were calculated by unconditional logistic regression and utilized to evaluate the potential associations between RECQL5 genetic variants and OS risk. Statistical significance was established at $\mathrm{P}<0.05$. 


\section{RESULTS}

We enrolled 185 OS patients and 201 healthy control subjects. The general characteristics of the 2 groups are shown in Table 1. There were no significant differences between OS patients and cancer-free controls in gender, age, tumor location, and metastasis.

\begin{tabular}{|c|c|c|c|}
\hline Characteristics & Cases [N (\%)] & Controls [N (\%)] & $P$ value \\
\hline Number & 185 & 201 & \\
\hline Gender (male) & $97(52.4)$ & $101(50.2)$ & 0.634 \\
\hline Age (years) & $32.3 \pm 12.1$ & $33.1 \pm 12.4$ & 0.565 \\
\hline Tumor location & & - & \\
\hline Long tubular bones & $121(65.4)$ & - & \\
\hline Axial skeleton & $64(34.6)$ & - & \\
\hline Metastasis & & - & \\
\hline Yes & $84(45.4)$ & - & \\
\hline No & $101(54.6)$ & - & \\
\hline
\end{tabular}

The genotype distribution showed no significant difference from Hardy-Weinberg equilibrium values (data not shown). We found that the CC genotype in rs820196 was more frequent in the OS group than in the control group. We also found that the $\mathrm{C}$ allele of rs820196 was more common in the $\mathrm{OS}$ patients than in control subjects $(\mathrm{OR}=1.87,95 \% \mathrm{CI}=1.21-1.97$; $\mathrm{P}=0.001 ;$ Table 2).

Table 2. Genotype distribution of RECQL5 SNP between cases and controls.
\begin{tabular}{lccccc}
\hline SNPs & Genotype and allele & Osteosarcoma (N = 185) & Control $(\mathrm{N}=201)$ & OR $(95 \% \mathrm{CI})$ & P value \\
\hline rs820196 & CC & $30(0.162)$ & $18(0.090)$ & & 0.005 \\
& CT & $96(0.519)$ & $102(0.507)$ & & \\
& TT & $59(0.319)$ & $81(0.403)$ & $1.87(1.21-1.97)$ & 0.001 \\
C & $156(0.422)$ & $138(0.338)$ & & \\
\hline
\end{tabular}

\section{DISCUSSION}

In this study, we found that the RECQL5 gene polymorphism was associated with OS risk in a Chinese population. Previous studies indicated that RECQL5 plays a role in DNA replication, DNA repair, homologous recombination, and RNA polymerase II-mediated transcription (Pellatt et al., 2012). Additionally, the RECQL5 polymorphism was reported to be associated with colon cancer (Pellatt et al., 2012). However, the relationship between the RECQL5 polymorphism and OS is unknown. To clarify the relationship between the RECQL5 genetic polymorphism and OS, we performed a case-control study to genotype 1 single nucleotide polymorphism in the RECQL5 gene in a Chinese population. We found that the genotype distribution for rs820196 was significantly different between OS patients and control subjects. This suggested that subjects with the $\mathrm{C}$ allele of rs820196 increased the susceptibility to OS.

There were several limitations to this study. The sample size was relatively small, which may have affected the power of the statistical analysis; additionally, we did not perform a functional study to examine the mechanism of how genetic polymorphisms in RECQL5 affect OS risk. 
In conclusion, our findings suggest that $\mathrm{OS}$ is associated with the RECQL5 gene polymorphism in a Chinese Han population.

\section{ACKNOWLEDGMENTS}

Research supported by the Scientific Research Fund of Xinxiang Medical University and Science and Technology Innovation Talents in Universities in Ministry of Education of Henan Province in 2010 (\#2010HASTIT036).

\section{REFERENCES}

Bohr VA (2008). Rising from the RecQ-age: the role of human RecQ helicases in genome maintenance. Trends Biochem. Sci. 33: 609-620.

Dorfman HD and Czerniak B (1995). Bone cancers. Cancer 75: 203-210.

Hu Y, Raynard S, Sehorn MG, Lu X, et al. (2007). RECQL5/Recq15 helicase regulates homologous recombination and suppresses tumor formation via disruption of Rad51 presynaptic filaments. Genes Dev. 21: 3073-3084.

Kager L, Zoubek A, Potschger U, Kastner U, et al. (2003). Primary metastatic osteosarcoma: presentation and outcome of patients treated on neoadjuvant Cooperative Osteosarcoma Study Group protocols. J. Clin. Oncol. 21: 2011-2018.

Li D, Suzuki H, Liu B, Morris J, et al. (2009). DNA repair gene polymorphisms and risk of pancreatic cancer. Clin. Cancer Res. 15: 740-746.

Mirabello L, Troisi RJ and Savage SA (2009). Osteosarcoma incidence and survival rates from 1973 to 2004: data from the Surveillance, Epidemiology, and End Results Program. Cancer 115: 1531-1543.

Pellatt AJ, Wolff RK, Lundgreen A, Cawthon R, et al. (2012). Genetic and lifestyle influence on telomere length and subsequent risk of colon cancer in a case control study. Int. J. Mol. Epidemiol. Genet. 3: 184-194.

Sharma S, Doherty KM and Brosh RM Jr (2006). Mechanisms of RecQ helicases in pathways of DNA metabolism and maintenance of genomic stability. Biochem. J. 398: 319-337.

Wang L, Liu Z, Jing P, Shao L, et al. (2014). Effects of murine double minute 2 polymorphisms on the risk and survival of osteosarcoma: a systemic review and meta-analysis. Tumour Biol. 35: 1649-1652.

Zhou Y, Liu B, Wang M and Ni J (2014). Endothelin-1 gene polymorphisms and risk of chemoresistant pediatric osteosarcoma. Pediatr. Blood Cancer 61: 612-617. 